\title{
2D rigid benzoxazole-linked covalent organic framework films with high-strength, high- modulus mechanical behavior
}

\author{
Kristen A. Miller ${ }^{\dagger}$ Lawrence B. Alemany, ${ }^{\ddagger}$ Edwin L. Thomas $*, \dagger$ and Eilaf Egap ${ }^{*, \dagger}$ \\ ${ }^{\dagger}$ Department of Materials Science and NanoEngineering, Rice University, Houston, TX 77005, United States \\ ${ }^{\ddagger}$ Department of Chemistry and Shared Equipment Authority, Rice University, Houston, TX 77005, United States
}

\begin{abstract}
Two-dimensional (2D) benzoxazole-linked covalent organic frameworks (COFs) provide an opportunity to incorporate the strength and modulus of corresponding $1 \mathrm{D}$ rigid-rod polymers into multiple directions by extending covalent bonding into two dimensions while simultaneously reducing density. Thus far, this potential has been elusive because of the challenge of producing high-quality COF films, particularly those with irreversible, rigid benzazole linkages. The majority of COF syntheses use a single-step process approach where polymerization occurs faster than crystallization and typically result in a poorly ordered and insoluble powder. Here, we present a one-step synthesis and two-step process that allows the deposition of a uniform intermediate film via reversible, non-covalent interactions. This network then undergoes an annealing step that facilitates the irreversible conversion to 2D covalently-bonded polymer product. The resulting films are semi-crystalline with platelet-like crystals embedded in an amorphous matrix with sharp crystal-amorphous interfaces. By this approach, we achieve free-standing films for which we demonstrate the first example of mechanical testing of benzazole-linked COFs. These initial films have promising mechanical properties with an in-plane ultimate tensile strength of nearly $50 \mathrm{MPa}$ and axial tensile and transverse compressive elastic moduli on the scale of several GPa. These mechanical properties already rival the performance of solution-cast films of $1 \mathrm{D}$ polybenzoxazole (PBO).
\end{abstract}

\section{INTRODUCTION}

Covalent organic frameworks (COFs) are an emerging category of materials that extend traditional one-dimensional polymer chemistry into two or three dimensions to produce nano-porous networks linked entirely by covalent bonds. COFs have attracted much attention because of their favorable properties such as permanent porosity, high surface area, low density, tailorable structure and stability under harsh conditions. ${ }^{1-}$

${ }^{7}$ While much progress has been made regarding synthetic techniques ${ }^{3,4,6,8-11}$ and expanding into novel structures and linkages $7,12-22$, the study of intrinsic material properties and implementation into various applications remains limited by the challenge of producing highquality, highly-crystalline, mechanically robust $\mathrm{COF}$ films. Conceptually, translating the connectivity of onedimensional (1D) polymers into $2 \mathrm{D}$ to create graphenelike structures would provide enhanced charge transport and mechanical strength in comparison to their 1D counterparts that are limited by non-covalent lateral interactions between polymer chains. ${ }^{23-25}$ Additionally, COFs are nanoporous materials and thus expected to be low-density materials compared to $1 \mathrm{D}$ polymeric structures. In this report, we choose to focus on the benzobisoxazole linkage because their respective rigid 1D polymers, specifically PBO or Zylon, exhibit exceptional mechanical properties and are considered among the highest strength polymers. Our motivation comes from the potential to produce functional materials that have a similar strength and modulus to PBO but across a 2D plane, while also decreasing the overall density.

To date, the most common method for synthesizing COF materials remains a solvothermal reaction in which a flame-sealed tube is heated for several days under vacuum or inert gas. This method typically yields a powder that is insoluble in common organic solvents and acids. These single-step methods are thermodynamically controlled where bond formation occurs quickly, followed by slow reorganization into a lower-energy structure with limited order ${ }^{26}$ as evidenced by the presence of only a few broad diffraction peaks. While films of 2D and 3D COFs have been previously synthesized using methods such as solvothermal synthesis, interfacial polymerization and vapor-assisted conversion $^{27}$, major limitations include difficulty of scaleup, the requirement of a support, long reaction times and low yield of starting material converted to actual film.

Benzoxazole-linked COFs have been synthesized previously using different synthetic strategies including the traditional sealed-tube solvothermal method $^{7,9,28}$ but unfortunately, the product is an insoluble powder. Here, 
we present a novel one-step solvothermal synthesis that involves a two-step processing method, in which a network is formed through dynamic bond interactions, followed by irreversible conversion to polymer product. The molecular structure of the final COF product was confirmed by solid-state nuclear magnetic resonance spectroscopy (NMR), Fourier-transform infrared spectroscopy (FTIR) and Raman spectroscopy and we propose that this reaction proceeds via a different mechanism through an oxidized quinone intermediate, as evidenced by characterization of the intermediate films. This bottom-up approach produces free-standing films of benzoxazole-linked COFs on the scale of several square centimeters within 24 hours. The stability of the intermediate films and the COF product free-standing films was examined in strong acid and base solutions. We find that product films remained stable and intact with no apparent dissolution of material even in strong acids such as methanesulfonic acid. In contrast to the synthesis of powder benzoxazole COFs, the synthetic method we report here proceeds via a different mechanism and takes advantage of a dynamic intermediate to facilitate a unique crystallization of the COF films with exceptional order. The micro- and macroscopic morphology is elucidated using polarized optical microscopy, atomic force microscopy (AFM) and scanning electron microscopy (SEM) and reveal rod features embedded within the films with birefringence observed in both the rods and the uniform film matrix. Transmission electron microscopy (TEM) allows us to investigate the microstructure of these COF films and reveals a two-phase semi-crystalline structure with amorphous material surrounding welldefined, faceted crystal platelets. Moreover, these freestanding films already show promising mechanical properties through tensile testing and nanoindentation.

\section{RESULTS AND DISCUSSION}

\section{Synthesis:}

The benzobisoxazole-linked TFPPy-BBO-COF reported herein (initially referred to as "LZU-192" in powder form $)^{7}$ can be synthesized using a one-step synthesis, two-step processing approach as shown in Figure 1a. First, a dilute reaction mixture is made by combining 1,3,6,8-tetrakis-(4-formylphenyl)pyrene (TFPPy) and 2,5-diaminohydroquinone dihydrochloride (DAHQ) monomers along with benzimidazole catalyst in a solvent mixture of $1: 1 \mathrm{v} / \mathrm{v} \mathrm{N}$-methyl-2-pyrrolidone (NMP) and mesitylene. The solution is drop-cast onto a substrate in air at ambient pressure and the temperature is ramped from room temperature to $100^{\circ} \mathrm{C}$ (Figure S1) to slowly evaporate the solvent. As the temperature increases, there is a distinct color change from the initial yellow monomer mixture to a transparent orange solution, indicating that the initial reaction occurs within minutes. After the solvent has fully evaporated over the course of about 30 minutes, an opaque orange/brown film remains. In the second step, the film is annealed at $200^{\circ} \mathrm{C}$ under vacuum, where the intermediate film is irreversibly converted to the final benzoxazole-linked COF structure in the form of a dark brown film that is $2-3 \mu \mathrm{m}$ thick and can be easily removed from the glass substrate. Alternatively, if good adherence to the substrate is desired rather than free-standing form, this can be achieved by simply performing a UV-ozone treatment of the substrate beforehand. During the annealing process, the benzimidazole is evaporated off and therefore this method does not require subsequent purification to remove the catalyst.

To understand the reaction mechanism of TFPPyBBO-COF film formation, we investigated the chemical composition of both the intermediate film and the final annealed film. We hypothesize that the film formation of TFPPy-BBO-COF proceeds via a different reaction mechanism compared to previous reports of benzoxazolelinked COFs, ${ }^{7,28}$ particularly given that the first step of our procedure is performed in air. Our proposed mechanism is illustrated in Figure 1b, where the DAHQ monomer is initially oxidized to the $p$-benzoquinone form (DABQ), which then undergoes a condensation reaction with the aldehyde groups on TFPPy to form a reversible imine-linked intermediate. Finally, dehydrogenation results in irreversible ring-closure to form the benzobisoxazole. Generally, the DAHQ monomer presents a solubility issue in reaction mixtures, however, the DABQ form is readily soluble in the NMP/mesitylene co-solvent, which likely assists in obtaining a faster and more complete conversion to product. 


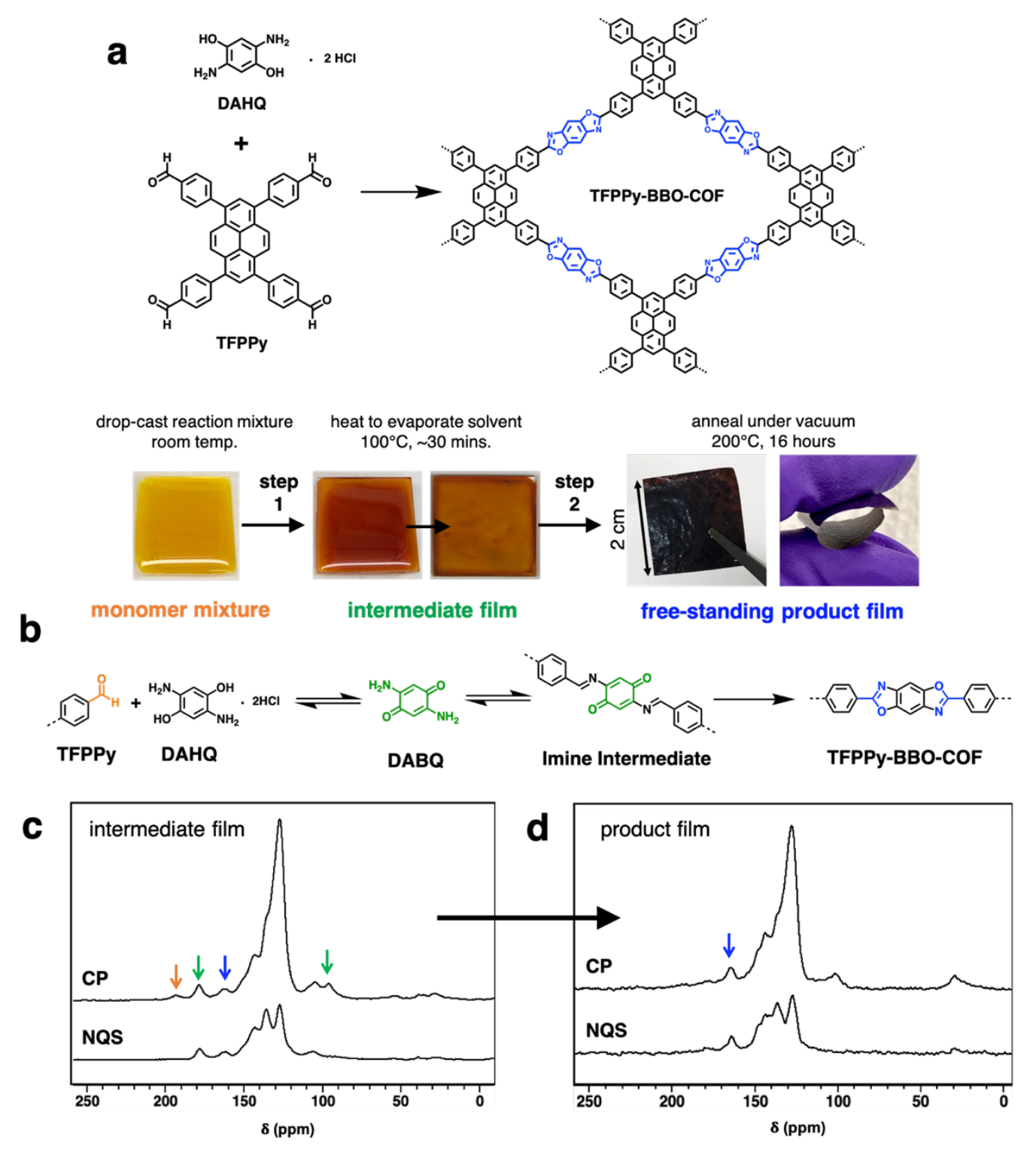

Figure 1. TFPPy-BBO-COF Film Synthesis.

a. Schematic showing reactant and product structures along with photographs depicting the two-step synthesis process for the formation of free-standing TFPPy-BBO-COF films. b. Proposed mechanism for the formation of the benzoxazole linker. c. Solid-state ${ }^{13} \mathrm{C}$ NMR spectra show that the intermediate film contains a mixture of unreacted monomer (194 ppm), intermediate $(178,96 \mathrm{ppm})$ and product $(163 \mathrm{ppm})$ and $\mathbf{d}$. after annealing the films are converted entirely to benzoxazole product.

Solid-state ${ }^{13} \mathrm{C}$ nuclear magnetic resonance (NMR) spectroscopy experiments allow analysis of the composition of the films at the different steps in the synthesis. As expected, the cross-polarization/magic angle spinning (CP/MAS) ${ }^{13} \mathrm{C}$ NMR spectrum of the intermediate film (Figure 1c) differs significantly from that of the final product ${ }^{7}$ with contrasting peaks appearing at 194, 178 and $96 \mathrm{ppm}$. In the non-quaternary suppression (NQS) experiment, the peaks at $194 \mathrm{ppm}$ and
$96 \mathrm{ppm}$ decay completely, indicating that these signals originate from non-quaternary carbons. The peak at 194 ppm is consistent with the chemical shift of the aldehyde carbon from unreacted TFPPy monomer (Figure S5). The peaks at $178 \mathrm{ppm}$ and $96 \mathrm{ppm}$ are assigned to the DABQ intermediate (Figure S6). ${ }^{29}$ Lastly, the signature oxazole quaternary carbon peak at $163 \mathrm{ppm}$ appears in the intermediate film, suggesting that there is already some final product formed. The solid-state ${ }^{13} \mathrm{C}$ NMR results of 
the intermediate film show a mixture of unreacted monomer, intermediate and final product. Since different carbons cross polarize at different rates, we are unable to quantitatively estimate a percent conversion of the intermediate film. Additionally, the aldehyde monomer signal is underrepresented due to washing steps in order to remove the benzimidazole catalyst from the intermediate samples since they are not annealed (see SI for more details).

The CP/MAS NMR spectrum of the final product film (Figure 1d) confirms the structure of benzoxazole product. The standard CP and CP with NQS spectra are clearly consistent with those previously reported for the powder form. ${ }^{7,9}$ The highlighted peak at a chemical shift of $164 \mathrm{ppm}$ is attributed to the substituted C-2 carbon of a 1,3-oxazole ring and confirms the formation of new bonds between the small molecule building blocks. Furthermore, the signal at $164 \mathrm{ppm}$ in the NQS experiment, remains at full intensity, confirming a quaternary carbon. As with the intermediate film, this signal cross polarizes relatively slowly in a series of experiments with different contact times. Additional peak assignments are provided in the SI (Figure S3). Finally, the solid-state ${ }^{13} \mathrm{C}$ NMR clearly shows that the annealed film consists solely of benzoxazole product with no starting materials.

Fourier Transform Infrared Spectroscopy (FTIR) is also used to characterize the chemical composition of the intermediate film and COF product film (Figure S7). We performed a series of varied anneal times, starting from no anneal then 4,8 , and 16 hours of annealing at $200^{\circ} \mathrm{C}$ under vacuum. From this series, we can observe the gradual emergence of a peak at $1603 \mathrm{~cm}^{-1}$ that is attributed to the $-\mathrm{C}=\mathrm{N}$ - stretching in the oxazole heterocycle. This peak can be clearly distinguished from the aromatic $-\mathrm{C}=\mathrm{C}$ - bending at $1577 \mathrm{~cm}^{-1}$. Because of the proximity of these two peaks, it is sometimes difficult to distinguish the $-\mathrm{C}=\mathrm{N}$ - signal, which is evident in previous work by Waller et al. for the same structure. ${ }^{9} \mathrm{We}$ also identify a peak at $1119 \mathrm{~cm}^{-1}$ that can be attributed to the C-O- stretching within the oxazole ring. ${ }^{7,9,30}$ These FTIR results support the findings of the solid-state NMR experiments and allow us to not only identify the formation of oxazole product, but also confirm the absence of starting material.

Our hypothesized reaction mechanism through the DABQ intermediate is consistent with small molecule synthesis of benzobisoxazoles starting from the DABQ monomer ${ }^{31}$ as well as our ability to make TFPPy-BBOCOF directly from the $p$-benzoquinone form (Figure S9). Interestingly, the films made directly from the DABQ monomer result in small flakes rather than a single continuous film, indicating there is some benefit to oxidizing the hydroquinone form simultaneously with the condensation reaction, rather than performing the oxidation step beforehand.

A series of acid and base stability tests can be performed to support the formation of the ultra-stable benzoxazole-linked polymer product. Both intermediate films and product films were soaked in neat hydrochloric acid $(\mathrm{HCl})$, methanesulfonic acid (MSA) and $9 \mathrm{M} \mathrm{NaOH}$ for 16 hours. In all cases, the product films remained stable and intact with no apparent dissolution of material into the acid or base solutions (Figure S10). We can confirm the stability by collecting FTIR spectra before and after the treatments (Figure S11). In comparison, during acid treatments of the intermediate films, a distinct color change in the solution is observed, indicating dissolution of starting material and/or reversibly-linked intermediate. In MSA, the intermediate film breaks up and almost entirely dissolves. Additionally, the acid treatments result in a reversible color change to a red hue for both the intermediate and product films, indicating reversible protonation of the nitrogen's in the oxazole ring.

A curious feature observed in these films is the presence of rod-shaped areas that are roughly 20-30 $\mu \mathrm{m}$ in length and about $2 \mu \mathrm{m}$ wide embedded within the film (Figures S12-16). The formation of similar rods has been previously observed both for the TFPPy-BBO-COF in powder form as well as similar COF structures that also use the TFPPy monomer, even though the crystal structure varies. $^{7,} 19,32,33$ SEM reveals that the rods have sharp edges and planar surfaces that resemble facets, suggesting single crystals (Figure S13). We hypothesize that for our process, the rod features are initially formed from the ordering of the reversibly-linked building blocks and directed by the pyrene-based monomer units, considering the TFPPy monomer alone forms rod-shaped crystals. As the intermediate film is converted to irreversibly-linked polymer in the solid-state, the macroscopic form of the rod is preserved. This preservation of monomer crystal faceted morphology has been reported in solid-state polymerizations. ${ }^{34,}{ }^{35}$ From polarized optical microscopy, the rods appear to have birefringent characteristics along with areas of film between rods (Figure S15).

Using Raman spectroscopy, we can collect spectra for the intermediate and product films directly on the rod-shaped features (Figure S17). The presence of sharp, intense Raman signals from both the intermediate and product exposed rods indicate that these are highly 
crystalline regions. Comparing to the spectrum for TFPPy, we can see many contrasting peaks, suggesting that the rods are not simply unreacted monomer crystals. In the TFPPy spectrum, we identify key peaks at 1178 , 1386 and $1703 \mathrm{~cm}^{-1}$ that are attributed to vibrations resulting from the aldehyde functionality. In the product film rods, we identify a peak at $1572 \mathrm{~cm}^{-1}$ that can be assigned to the oxazole $\mathrm{C}=\mathrm{N}$ stretching ${ }^{36}$. Further details of peak assignments are provided in the SI.

In order to gain insight into the surface area and porosity of the COF films, gas adsorption experiments were performed using both $\mathrm{N}_{2}$ and $\mathrm{CO}_{2}$ gases. From the $\mathrm{N}_{2}$ isotherm (Figure S18), we can see a small degree of micropore filling and stepwise character in both the adsorption and desorption curves. This may be due to unfavorable interactions between our films and the adsorbate molecules. From this experiment we can calculate the surface area for the films to be $36.4 \mathrm{~m}^{2} / \mathrm{g}$. In contrast, when $\mathrm{CO}_{2}$ is used as the adsorbate, we observe a more regular isotherm (Figure S19) with a higher calculated surface area of $76.7 \mathrm{~m}^{2} / \mathrm{g}$, although this is still relatively low for COFs. The low calculated surface areas could be attributed to the thin film morphology, semicrystalline microstructure, or a staggered-type order as opposed to an eclipsed formation with channel pores. In both types of experiments, the pore size distribution is fairly consistent, indicating large pores with a distribution maximum at $27.7 \AA$.

\section{Crystal Structure:}

Transmission electron microscopy (TEM) and electron diffraction were used to observe the nano-scale structure of the as-synthesized COF films in order to gain structural insight. Generally, specimen areas are too thick for high-resolution TEM imaging, however thin edge areas of fractured pieces can be observed as well as regions in thinner films made from a diluted reaction mixture. The low-magnification bright-field TEM micrograph in Figure 2a shows a two-phase morphology of the films with platelet-like crystals embedded in an amorphous matrix (see SI for more details). The crystals have facets that often intersect at angles of about 55, 70 or 110 degrees. As mentioned earlier, we observe microscopic rod features embedded in the films. From low-magnification TEM, however, we can see that the size of the rods is almost 2 orders of magnitude larger than the polymer crystal platelet size (Figure S21). We also observe that the semi-crystalline nature of the polymer product is not only located in the rod features but within the uniform film matrix as well. For this semicrystalline morphology, the amorphous region encapsulating crystallites allows the formation of a uniform flexible, macroscopic film.

Through electron microscopy, we are able to elucidate the complex structural features of these COF films on both the micro- and nano-scale down to the unit cell (Figure 3). The TFPPy-BBO-COF material is very resistant to beam damage, allowing acquisition of lattice images and diffraction patterns. Additionally, as with the crystals, the amorphous region is not easily damaged by the electron beam. We hypothesize that the amorphous region is formed from nonplanar connectivity of the COF structure while maintaining the same chemical composition and covalent linkages. Given that the lateral dimensions of the crystallites are on the scale of several 1,000 's of Angstroms, it is easy to isolate a crystal using a selected-area diffraction (SAD) aperture. SAD patterns were obtained using a double-tilt holder to produce patterns from zone axes. The SAD pattern in Figure 2c shows a large array of sharp, intense reflections, which indicates exceptional periodic order within this crystal. Figure $2 b$ shows a single crystal area from within one of the platelets with well-resolved lattice fringes throughout the entire image. Such order is rarely observed thus far for COFs, and especially for 2D COFs. ${ }^{11,37}$ For comparison, the TFPPy monomer crystals are observed using TEM in Figure S24. Unlike the COF crystal platelets, the TFPPy crystals display a ribbon-like morphology with a corresponding selected-area diffraction pattern. The intensity of the pattern dissipates within seconds of beam exposure and no lattice images could be obtained due to beam damage of the small molecule crystals. This indicates that the crystals we observe in the product material are not simply unreacted monomer crystals.

We designed a to-scale-schematic model of the COF molecular structure (Figure 2d) in order to simulate a diffraction pattern via a 2D Fast Fourier Transform (FFT). The model FFT in Figure 2e indicates a basecentered structure where $b>a$. Allowed reflections appear for indices where $\mathrm{h}+\mathrm{k}=2 \mathrm{n}$ with the most intense being the diagonal reflections corresponding to the $\begin{cases}1 & 1\end{cases}$ $0\}$ family. The experimental SAD pattern corresponds well a based-centered unit cell, except for the scale of the main reflections (d-spacings of 3.2, 2.7 and $2.0 \AA$ ) are at q's that are a factor $6 x$ larger, indicating that these are high order reflections ( ( $\left.\begin{array}{lll}6 & 6 & 0\end{array}\right),\left(\begin{array}{lll}0 & 12 & 0\end{array}\right)$ and $\left(\begin{array}{lll}12 & 0 & 0\end{array}\right)$ respectively). The experimental FFT of the multibeam lattice image in Figure $2 \mathrm{f}$ shows a very similar pattern, 

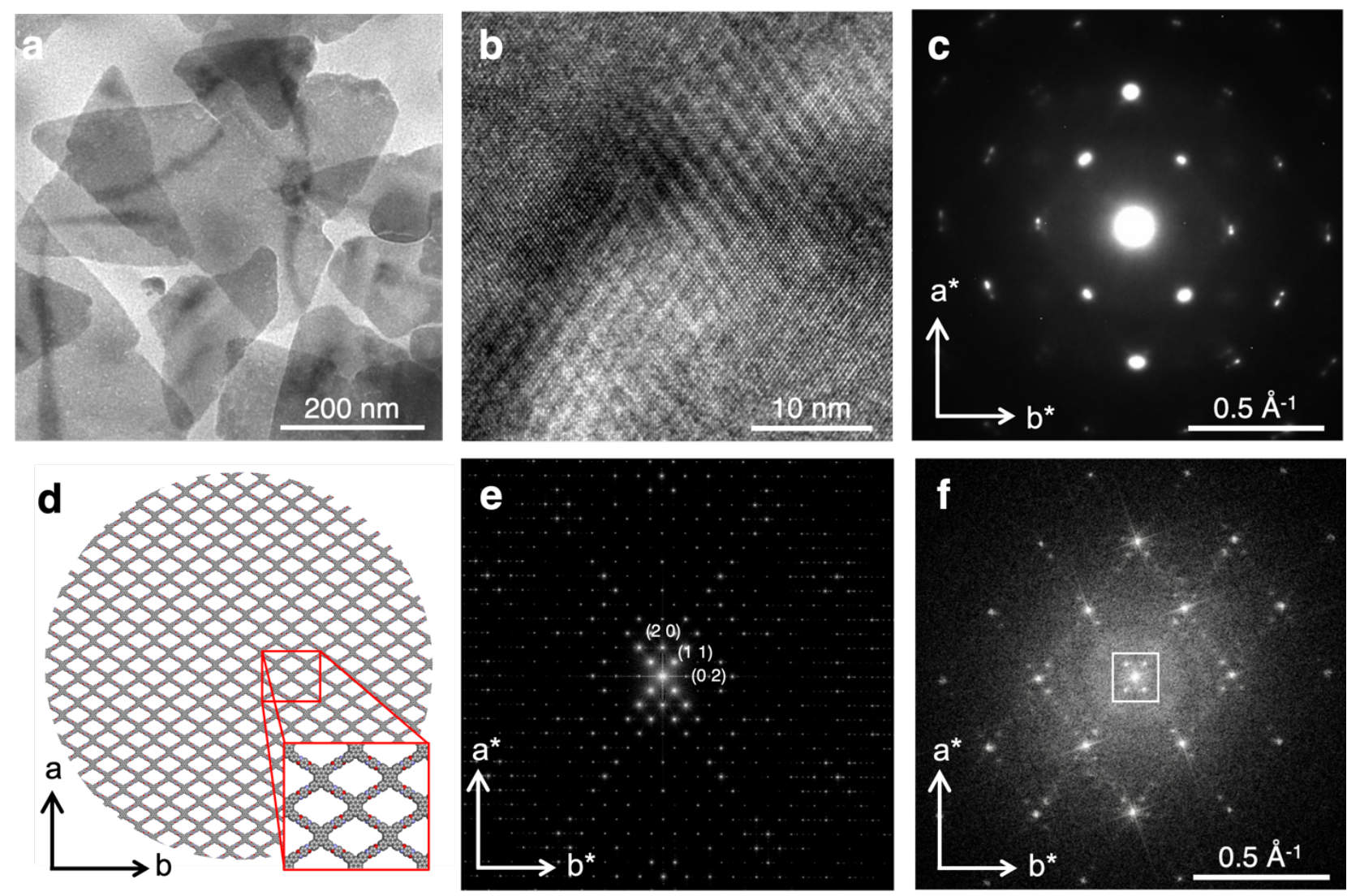

Figure 2. TEM and Electron Diffraction Analysis of Single Crystal TFPPy-BBO-COF Platelets.

a. Low-magnification BF TEM micrograph of a thin film region showing platelet-like crystals (triangular regions, some containing dark bend contours) surrounded by the lighter amorphous matrix material. b. High-resolution BF TEM micrograph from within a crystal with lattice fringes throughout the image. c. Selected-area $\left\{\right.$ hk0\} electron diffraction pattern from the $\left[\begin{array}{lll}0 & 0 & 1\end{array}\right]$ zone axis. d. Molecular model of a single layer of the base-centered TFPPy-BBO-COF structure and e. the simulated 2D FFT of this model. f. Experimental FFT of BF multi-beam lattice image shows large d-spacing features that match well with the simulated FFT pattern in e.

moreover, we are able to additionally resolve 2 pairs of quite large d-spacing spots corresponding to a spacing of $20 \AA$. Comparing the FFT of the lattice image to the FFT of the model, these intense reflections at $20 \AA$ match well with the $\left\{\begin{array}{lll}1 & 1 & 0\end{array}\right\}$ family in our proposed unit cell. These low order reflections are not resolved in the SAD pattern most likely due to their low relative intensity and proximity to the bright central $\left(\begin{array}{lll}0 & 0 & 0\end{array}\right)$ spot. Indexing of the SAD and FFT patterns is included in the SI. The angles between $\left\{\begin{array}{lll}1 & 1 & 0\end{array}\right\}$ reflections in the SAD pattern and experimental FFT are measured at 70 and 110 degrees, which is consistent with the angles between major facets observed at the edges of the crystal platelets.

\section{Mechanical Properties:}

Tensile testing and nanoindentation techniques demonstrate highly-promising mechanical properties of the TFPPy-BBO-COF films. From tensile testing experiments, fracture of the film typically results in a single macroscopic crack propagating across the width of the specimen and normal to the applied force (Figure 4a). Through cross-sectional SEM imagining, we observe the formation of voids on the fracture surface (Figure S25). The ultimate tensile strength (UTS) is calculated to be 48 $\pm 8 \mathrm{MPa}$ (Figure 4b). The strain at fracture varied considerably from sample to sample most likely due to flaws and defects that are unique to individual film pieces (see Figure S26). The measured stress-strain curves take on a J-shape with a concave upward region before reaching the linear elastic region. This behavior has been observed for rehydrated collagen fibers ${ }^{38}$ where, as the material extends, the lower modulus components elongate initially and the fibrils reorient in the direction of the applied force, resulting in a stiffening of the polymer. ${ }^{38,39}$ The initial low modulus toe region in the TFPPy-BBOCOF films is likely due to the deformation of the 

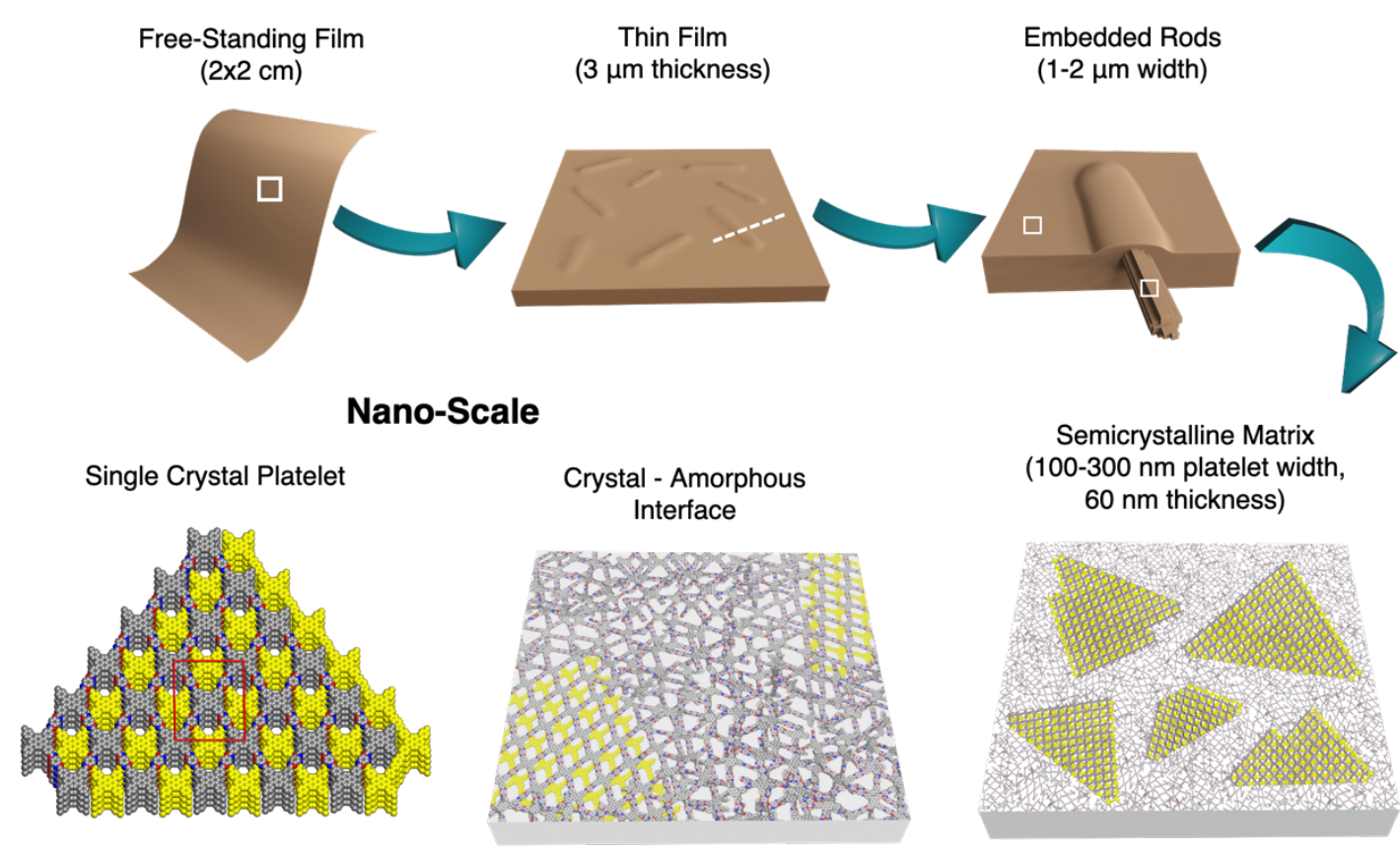

Semicrystalline Matrix (100-300 nm platelet width, $60 \mathrm{~nm}$ thickness)

Figure 3. TFPPy-BBO-COF Film Structure.

Schematic summarizing the complex structural hierarchy that exists within the films from the macro-scale to unit cell. The material consists of crystalline platelets embedded in an amorphous matrix.

amorphous regions between crystals that then undergo strain hardening. The resulting $2 \%$ strain in-plane elastic modulus is $2.6 \pm 1.5 \mathrm{GPa}$.

Nanoindentation experiments were conducted for displacements ranging from $40-800 \mathrm{~nm}$ on films 3 microns thick. We found for these samples that hardness values are reliable above a displacement of about $120 \mathrm{~nm}$ (Figure S27). Beyond $250 \mathrm{~nm}$, however, modulus values begin to increase with depth due to interference from substrate interaction. Generally, a good rule of thumb for nanoindentation is to use an indentation depth of about $10 \%$ of the film thickness. ${ }^{40}$ The displacement range we defined from these experiments is consistent with this standard. The loading and unloading curves indicate a highly elastic material as there is little hysteresis and minimal, if any, plastic deformation observed (Figure 4c). The average hardness was $1.15 \pm 0.08 \mathrm{GPa}$ with a compressive reduced elastic modulus of $10.6 \pm 0.6 \mathrm{GPa}$. In order to quantify elastic modulus from nanoindentation, knowledge of the Poisson's ratio of the material is required to convert from the reduced elastic modulus value. Another important factor is the semicrystalline nature of the material as well as the anisotropic shapes of the COF crystals such that the Poisson's ratio and elastic modulus would vary based on the direction in which the force is applied. Since the mechanical properties of this material are heretofore unexplored, there are, as yet, no reported values for an experimental or theoretical Poisson's ratio.

The strongest materials made from rigid-rod benzazole derivatives, such as the commercial Zylon (poly(p-phenylene-2,6-benzobisoxazole)), are spun from liquid crystalline solution into highly oriented fibers in order to greatly enhance axial mechanical performance. ${ }^{41}$

${ }^{43}$ The mechanical properties of our 2D TFPPy-BBO-COF thin films fall far short of such 1D PBO fibers, however, our film properties already rival those of solution-cast planar isotropic PBO films, which have UTS values ranging between $40-135 \mathrm{MPa}$ and tensile elastic moduli between 2-6 GPa. ${ }^{44-49}$ Nanoindentation studies for vapordeposited-polymerized as well as solution-cast thin films of PBO reported a compressive elastic modulus of about 11-12 GPa and a hardness of $\sim 0.5-0.7 \mathrm{GPa}^{50}$ Since the crystal density of the COF structure would theoretically be lower than that of PBO fibers ${ }^{51}$, this means that the 2Dlinked benzoxazole COF with comparable strength and 
modulus to PBO films offers an advantage when considering density-normalized specific properties.
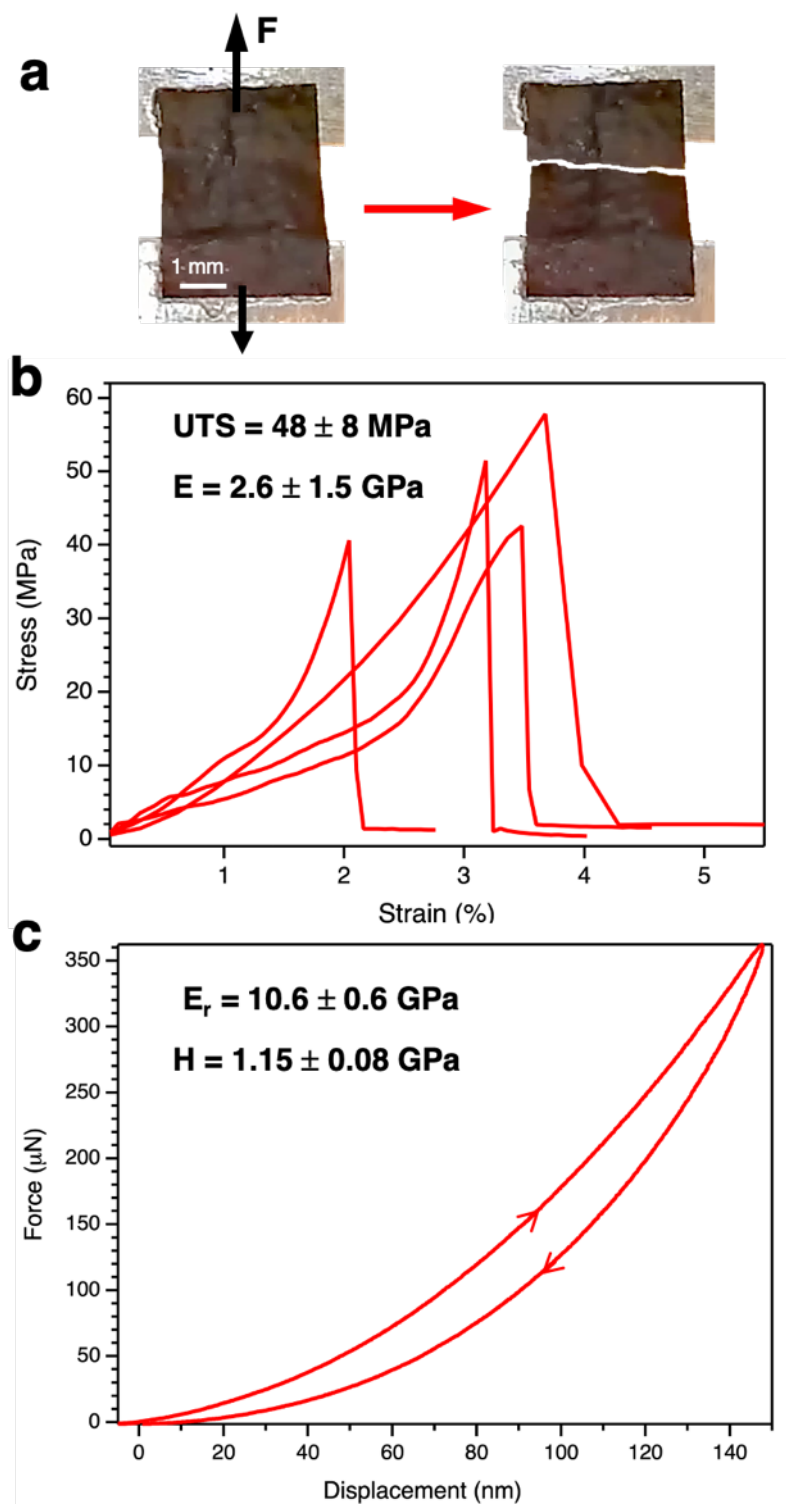

Figure 4. Mechanical Testing of COF Films.

a. Photographs of a film sample before and after tensile fracture. b. Stress-strain curves from tensile testing of film strips. c. Nanoindentation compressive loading and unloading curve shows highly elastic behavior.

\section{CONCLUSION}

Semi-crystalline thin films of benzoxazole-linked COFs were developed using a facile two-step processing approach, to take advantage of a unique mechanism in which the reaction proceeds through a series of reversible intermediate steps followed by irreversible conversion to final product. The initial solvent evaporation step occurs on a substrate gradually heated to $100^{\circ} \mathrm{C}$ where, as the reaction progresses and solvent leaves the system, the growing polymer platelets become confined to a $2 \mathrm{D}$ geometry. The films are then annealed under vacuum at $200^{\circ} \mathrm{C}$ to irreversibly convert the intermediate film entirely to benzoxazole-linked product. Analysis of the crystal structure using TEM and selected-area electron diffraction indicates an exceptional order within the material with faceted crystal platelets embedded in an amorphous matrix. Fast Fourier Transforms of highresolution TEM images of single crystals verify the inplane crystal structure. This study elucidates the complex hierarchical structure of COF films at the various length scales extending from the film micro-structure down to the unit cell. The ability to achieve free-standing films permitted the first example of mechanical testing of benzoxazole-linked COFs for both in-plane tension and transverse compression. These preliminary films exhibit impressive ultimate tensile strength and elastic moduli comparable to thin films of $1 \mathrm{D}$ PBO, warranting additional mechanical studies. Further development of synthesis and film processing to vary the degree of crystallinity and to enable growth of larger and interconnected crystals and improved film uniformity will bring us closer to being able to study the intrinsic properties of these materials.

\section{ACKNOWLEDGEMENTS}

The authors would like to acknowledge the National Science Foundation (NSF) for financial support under grant numbers CHE-1821863 (EE) and DMR1742864 (ELT). This work was conducted in part using resources of the Shared Equipment Authority (SEA) at Rice University. Thank you to Dr. Xueyan Feng for his help with modeling FFTs; to Prof. Jun Lou's group, especially Dr. Hua Guo and Boyu Zhang, for their help with the tensile testing instrumentation; and to Prof. Pulickel Ajayan's group, specifically Dr. Soumya Roy, for the helpful discussions. Additionally, thank you to Dr. Rahul Rao at the Air Force Research Laboratory, Materials and Manufacturing Directorate, for contributing Raman spectroscopy data and analysis. 


\section{Methods}

Materials. All commercial chemicals were used as purchased without further purification. 2,5-diaminohydroquinone dihydrochloride, benzimidazole, $\mathrm{N}$ methyl-2-pyrrolidone (NMP) and mesitylene were purchased from Sigma Aldrich. 1,3,6,8-Tetrakis-(4-formylphenyl)pyrene (TFPPy) was synthesized according to previously reported methods. ${ }^{19}$

TFPPy-BBO-COF Film Synthesis. 2,5-Diaminohydroquinone dihydrochloride $(5.4 \mathrm{mg}, 0.025 \mathrm{mmol}, 3.0 \mathrm{mg} / \mathrm{mL})$ and TFPPy $(7.8 \mathrm{mg}, 0.013$ $\mathrm{mmol}, 4.3 \mathrm{mg} / \mathrm{mL}$ ) were weighed into a vial with $0.9 \mathrm{~mL}$ of NMP and $0.9 \mathrm{~mL}$ of mesitylene then the mixture was sonicated for 15 minutes. Following sonication, benzimidazole $(6.6 \mathrm{mg}, 0.056 \mathrm{mmol}, 3.7 \mathrm{mg} / \mathrm{mL})$ was added to the vial and shaken to mix. The solution was immediately drop-casted $(250 \mu \mathrm{L})$ onto $2 \times 2 \mathrm{~cm}$ glass substrates or $1.8 \mathrm{~mL}$ onto $5 \times 5 \mathrm{~cm}$ substrates at room temperature. The substrates were then heated to $100^{\circ} \mathrm{C}$ until the solvent was fully evaporated (about 30 minutes), leaving behind an opaque orange/brown film. The film was then annealed under vacuum at $200^{\circ} \mathrm{C}$ for 16 hours to yield the final TFPPy-BBO-COF product in the form a dark brown film that delaminates from the substrate.

For analysis of intermediate film samples, following the drop-casting and solvent evaporation step, the films were treated with $\mathrm{N}, \mathrm{N}$-dimethylformamide (DMF) for one day followed by dichloromethane (DCM) for one day in order to remove benzimidazole and remaining solvent. The films were then transferred out of the DCM and dried on a hot plate at $70^{\circ} \mathrm{C}$ in air then further dried at room temperature under vacuum.

Solid-State ${ }^{13} \mathrm{C}$ Nuclear Magnetic Resonance (NMR). Experiments were performed using a $4.7 \mathrm{~T}\left(50.3 \mathrm{MHz}{ }^{13} \mathrm{C}\right.$ and $\left.200.1 \mathrm{MHz}{ }^{1} \mathrm{H}\right)$ Bruker spectrometer previously described in detail. ${ }^{52}{ }^{1} \mathrm{H}^{-13} \mathrm{C}$ cross-polarization/magic angle spinning (CP/MAS) experiments were performed with $7.6 \mathrm{kHz}$ MAS and a 5 second relaxation delay after the $32.8 \mathrm{~ms}$ FID. Contact times of $3.0 \mathrm{~ms}$ and $2.0 \mathrm{~ms}$ were used in the spectra shown for the TFPPy-BBO-COF films and intermediate films, respectively. To obtain additional information, experiments were performed with contact times of $0.2,0.5,0.9,1.4,2.0,3.0$, and $4.5 \mathrm{~ms}$ on the TFPPy-BBO-COF films and with contact times of $0.2,0.5$, $0.9,1.4$, and $2.0 \mathrm{~ms}$ on the intermediate films $(16,400$ scans for each spectrum). FIDs were processed with $50 \mathrm{~Hz}$ of line broadening. Nonquaternary suppression experiments were performed with a $25 \mu$ s dephasing delay before and after the $180^{\circ}{ }^{13} \mathrm{C}$ refocusing pulse. Chemical shifts are relative to glycine carbonyl at $176.46 \mathrm{ppm} .{ }^{53}$ In light of the limited amount of material available, the TFPPy-BBO-COF films were confined to the center of the rf coil region in the probe by using a $4 \mathrm{~mm}$-diameter rotor with a thickbottom and a removable silicon nitride upper plug. With a little more of the intermediate films available, a thick-bottom rotor was again used; there was not enough room to insert an upper plug.

Solution-State ${ }^{1} \mathbf{H}$ and ${ }^{13} \mathbf{C}$ NMR. Experiments were performed using a 500 $\mathrm{MHz}$ Bruker spectrometer, also described previously. ${ }^{52}$ The HSQC experiment was optimized for ${ }^{1} J_{\mathrm{CH}}=166.67 \mathrm{~Hz}$.

Fourier Transform Infrared (FTIR) spectra were obtained using a Nicolet iS50 FTIR spectrometer using a $\mathrm{KBr}$ detector and Smart iTX diamond ATR accessory. Data was collected within the range of $525-4000 \mathrm{~cm}^{-1}$.

Scanning Electron Microscopy (SEM) images were acquired using a FEI Helios NanoLab 660 DualBeam system operating at $1 \mathrm{kV}$ or $500 \mathrm{~V}$ with a current of $13 \mathrm{pA}$ in order to reduce charging.

Atomic Force Microscopy (AFM) data was acquired using a Park NX20 AFM operating in non-contact mode with Bruker TESPA-V2 antimony-doped silicon AFM tips.

Raman spectra were collected in an inVia Renishaw Raman microscope with an excitation wavelength of $633 \mathrm{~nm}$. For collecting spectra from the rods, a small $(\sim 5 \mathrm{~mm})$ portion of the COF films were placed on to a glass microscope slide and torn with a pair of tweezers. This resulted in the exposure of some of the rods. Spectra were collected (typically 4 acquisitions, $30 \mathrm{~s}$ each) from a 1 um-sized focused laser with a laser power of $100 \mathrm{uW}$ to ensure that the samples were not heated by the laser.

Gas Adsorption isotherms using $\mathrm{N}_{2}$ at $77 \mathrm{~K}$ and $\mathrm{CO}_{2}$ at $273 \mathrm{~K}$ were performed on the desolvated samples using a QUANTACHROME QUADRASORB-SI analyzer. About $50 \mathrm{mg}$ of each sample was degassed at $120^{\circ} \mathrm{C}$ under $10-1 \mathrm{~Pa}$ vacuum for about 12 hours before the measurements. $99.999 \%$ (UHP) pure helium gas was used to measure the dead volume of the sample cell.

Thermogravimetric Analysis (TGA) was conducted using a Mettler Toledo TGA/DSC 3+ instrument. Measurements were conducted under air or $\mathrm{N}_{2}$ atmospheres. Film samples were heated from $25^{\circ} \mathrm{C}$ to $800^{\circ} \mathrm{C}$ at a ramp rate of $5^{\circ} \mathrm{C} /$ min with a 30 minute isotherm at $100^{\circ} \mathrm{C}$ to remove water from the atmosphere.

Transmission Electron Microscopy (TEM) images and selected-area diffraction (SAD) patterns were acquired using a JEOL $2100 \mathrm{~F}$ with a field emission gun operating at $200 \mathrm{kV}$. A double-tilt sample holder was used to enable acquisition of zone axis patterns. For high-resolution images, a defocus value of $-44 \mathrm{~nm}$ was used. Selected-area aperture sizes of 10 and $20 \mu \mathrm{m}$ were inserted to isolate single crystals and collect electron diffraction patterns using camera lengths of 100 to $150 \mathrm{~cm}$.

Micro-Tensile Testing was performed using a Deben Microtest 200N device with a $2 \mathrm{~N}$ loadcell. The $\mathrm{COF}$ films were cut with a razor into rectangular strips approximately $3 \mathrm{~mm}$ wide and $5 \mathrm{~mm}$ long. Each end of the film strip was attached to a metal plate, which was then clamped in the instrument.

Nanoindentation was performed using a Hysitron TI 980 TriboIndenter equipped with a pyramidal diamond tip and calibrated using a polycarbonate standard sample. The experiments were conducted using a range of specified indentation depths from 30-1000 $\mathrm{nm}$ in order to identify a range of reliable hardness and elastic modulus measurements. 


\section{REFERENCES}

1. El-Kaderi, H. M.; Hunt, J. R.; Mendoza-Cortés, J. L.; Côté, A. P.; Taylor, R. E.; O'Keeffe, M.; Yaghi, O. M., Designed Synthesis of 3D Covalent Organic Frameworks. Science 2007, 316 (5822), 268-272.

2. Uribe-Romo, F. J.; Hunt, J. R.; Furukawa, H.; Klock, C.; O'Keeffe, M.; Yaghi, O. M., A crystalline imine-linked 3-D porous covalent organic framework. J. Am. Chem. Soc. 2009, 131, 4570-4571.

3. Matsumoto, M.; Dasari, R. R.; Ji, W.; Feriante, C. H.; Parker, T. C.; Marder, S. R.; Dichtel, W. R., Rapid, Low Temperature Formation of Imine-Linked Covalent Organic Frameworks Catalyzed by Metal Triflates. J. Am. Chem. Soc. 2017, 139, 4999-5002.

4. Vitaku, E.; Dichtel, W. R., Synthesis of 2D Imine-Linked Covalent Organic Frameworks through Formal Transimination Reactions. J. Am. Chem. Soc. 2017, 139, 12911-12914.

5. Karak, S.; Kandambeth, S.; Biswal, B. P.; Sasmal, H. S.; Kumar, S.; Pachfule, P.; Banerjee, R., Constructing Ultraporous Covalent Organic Frameworks in Seconds via an Organic Terracotta Process. J. Am. Chem. Soc. 2017, 139, $1856-$ 1862.

6. Biswal, B. P.; Chandra, S.; Kandambeth, S.; Lukose, B.; Heine, T.; Banerjee, R., Mechanochemical synthesis of chemically stable isoreticular covalent organic frameworks. J. Am. Chem. Soc. 2013, 135, 5328-5331.

7. Wei, P. F.; Qi, M. Z.; Wang, Z. P.; Ding, S. Y.; Yu, W.; Liu, Q.; Wang, L. K.; Wang, H. Z.; An, W. K.; Wang, W., Benzoxazole-Linked Ultrastable Covalent Organic Frameworks for Photocatalysis. J. Am. Chem. Soc. 2018, 140, 4623-4631.

8. Waller, P. J.; Lyle, S. J.; Osborn Popp, T. M.; Diercks, C. S.; Reimer, J.

A.; Yaghi, O. M., Chemical Conversion of Linkages in Covalent Organic Frameworks. J. Am. Chem. Soc. 2016, 138, 15519-15522.

9. Waller, P. J.; AlFaraj, Y. S.; Diercks, C. S.; Jarenwattananon, N. N.; Yaghi, O. M., Conversion of Imine to Oxazole and Thiazole Linkages in Covalent Organic Frameworks. J. Am. Chem. Soc. 2018, 140, 9099-9103.

10. $\quad$ Evans, A. M.; Parent, L. R.; Flanders, N. C.; Bisbey, R. P.; Vitaku, E.; Kirschner1, M. S.; Schaller, R. D.; Chen, L. X.; Gianneschi, N. C.; Dichtel, W. R., Seeded growth of single-crystal two-dimensional covalent organic frameworks. Science 2018, 361 (6397), 52-57.

11. Guo, X.; Tian, Y.; Zhang, M.; Li, Y.; Wen, R.; Li, X.; Li, X.; Xue, Y.; Ma, L.; Xia, C.; Li, S., Mechanistic Insight into Hydrogen-Bond-Controlled Crystallinity and Adsorption Property of Covalent Organic Frameworks from Flexible Building Blocks. Chem. Mater. 2018, 30, 2299-2308.

12. Xiang, Z.; Cao, D.; Huang, L.; Shui, J.; Wang, M.; Dai, L., Nitrogendoped holey graphitic carbon from $2 \mathrm{D}$ covalent organic polymers for oxygen reduction. Adv. Mater. 2014, 26, 3315-3320.

13. Liu, W.; Luo, X.; Bao, Y.; Liu, Y. P.; Ning, G. H.; Abdelwahab, I.; Li, L.; Nai, C. T.; Hu, Z. G.; Zhao, D.; Liu, B.; Quek, S. Y.; Loh, K. P., A twodimensional conjugated aromatic polymer via C-C coupling reaction. Nat. Chem. 2017, 9, 563-570.

14. Rager, S.; Jakowetz, A. C.; Gole, B.; Beuerle, F.; Medina, D. D.; Bein, T., Scaffold-Induced Diketopyrrolopyrrole Molecular Stacks in a Covalent Organic Framework. Chem. Mater. 2019, 31, 2707-2712.

15. Fu, Y.; Zhu, X.; Huang, L.; Zhang, X.; Zhang, F.; Zhu, W., Azine-based covalent organic frameworks as metal-free visible light photocatalysts for $\mathrm{CO} 2$ reduction with $\mathrm{H} 2 \mathrm{O}$. Applied Catalysis B: Environmental 2018, 239, 46-51.

16. Wan, S.; Gándara, F.; Asano, A.; Furukawa, H.; Saeki, A.; Dey, S. K.; Liao, L.; Ambrogio, M. W.; Botros, Y. Y.; Duan, X.; Seki, S.; Stoddart, J. F.; Yaghi, O. M., Covalent Organic Frameworks with High Charge Carrier Mobility. Chem. Mater. 2011, 23, 4094-4097.

17. Zhou, T. Y.; Xu, S. Q.; Wen, Q.; Pang, Z. F.; Zhao, X., One-step construction of two different kinds of pores in a $2 \mathrm{D}$ covalent organic framework. $J$. Am. Chem. Soc. 2014, 136, 15885-15888.

18. Wang, X.; Han, X.; Zhang, J.; Wu, X.; Liu, Y.; Cui, Y., Homochiral 2D Porous Covalent Organic Frameworks for Heterogeneous Asymmetric Catalysis. J. Am. Chem. Soc. 2016, 138, 12332-12335.

19. Rabbani, M. G.; Sekizkardes, A. K.; El-Kadri, O. M.; Kaafarani, B. R.; ElKaderi, H. M., Pyrene-directed growth of nanoporous benzimidazole-linked nanofibers and their application to selective $\mathrm{CO} 2$ capture and separation. J. Mater. Chem. 2012, 22, 25409-25417.

20. Guo, J.; Xu, Y.; Jin, S.; Chen, L.; Kaji, T.; Honsho, Y.; Addicoat, M. A.; Kim, J.; Saeki, A.; Ihee, H.; Seki, S.; Irle, S.; Hiramoto, M.; Gao, J.; Jiang, D., Conjugated organic framework with three-dimensionally ordered stable structure and delocalized pi clouds. Nat. Commun. 2013, 4, 2736.

21. Uribe-Romo, F. J.; Doonan, C. J.; Furukawa, H.; Oisaki, K.; Yaghi, O. M., Crystalline covalent organic frameworks with hydrazone linkages. J. Am. Chem. Soc. 2011, 133, 11478-11481.

22. Li, Z.; Feng, X.; Zou, Y.; Zhang, Y.; Xia, H.; Liu, X.; Mu, Y., A 2D azinelinked covalent organic framework for gas storage applications. Chem. Commun. 2014, 50, 13825-13828.

23. Zhang, P.; Ma, L.; Fan, F.; Zeng, Z.; Peng, C.; Loya, P. E.; Liu, Z.; Gong, Y.; Zhang, J.; Zhang, X.; Ajayan, P. M.; Zhu, T.; Lou, J., Fracture toughness of graphene. Nat. Commun. 2014, 5, 3782.

24. Sandoz-Rosado, E.; Beaudet, T. D.; Andzelm, J. W.; Wetzel, E. D., High strength films from oriented, hydrogen-bonded "graphamid" 2D polymer molecular ensembles. Sci. Rep. 2018, 8, 3708.

25. Sandoz-Rosado, E.; Wetzel, E. D., Mechanical Structure-Property Relationships for 2D Polymers Comprised of Nodes and Bridge Units. In Mechanics of Additive and Advanced Manufacturing, Volume 8, 2019; pp 25-27.

26. Colson, J. W.; Dichtel, W. R., Rationally synthesized two-dimensional polymers. Nat Chem 2013, 5 (6), 453-65.

27. Wang, H.; Zeng, Z.; Xu, P.; Li, L.; Zeng, G.; Xiao, R.; Tang, Z.; Huang, D.; Tang, L.; Lai, C.; Jiang, D.; Liu, Y.; Yi, H.; Qin, L.; Ye, S.; Ren, X.; Tang, W., Recent progress in covalent organic framework thin films: fabrications, applications and perspectives. Chem Soc Rev 2019, 48 (2), 488-516.

28. Pyles, D. A.; Crowe, J. W.; Baldwin, L. A.; McGrier, P. L., Synthesis of Benzobisoxazole-Linked Two-Dimensional Covalent Organic Frameworks and Their Carbon Dioxide Capture Properties. ACS Macro Lett. 2016, 5, 1055-1058.

29. Arani, M.; Bigdeli, N., Synthesis of Diamino Benzoquinones using Facile and One-Pot Chemical Oxidative Method. Oriental Journal of Chemistry 2016, 32 (6), 3135-3138.

30. El-Mahdy, A. F. M.; Hung, Y. H.; Mansoure, T. H.; Yu, H. H.; Chen, T.; Kuo, S. W., A Hollow Microtubular Triazine- and Benzobisoxazole-Based Covalent Organic Framework Presenting Sponge-Like Shells That Functions as a High-Performance Supercapacitor. Chem Asian J 2019, 14 (9), 1429-1435.

31. Osman, A.-M., Benzodioxazoles. J. Am. Chem. Soc. 1957, 79 (4), $966-$ 968.

32. Jin, E.; Li, J.; Geng, K.; Jiang, Q.; Xu, H.; Xu, Q.; Jiang, D., Designed synthesis of stable light-emitting two-dimensional sp2 carbon-conjugated covalent organic frameworks. Nature Communications 2018, 9 (1).

33. Jin, E.; Asada, M.; Xu, Q.; Dalapati, S.; Addicoat, M. A.; Brady, M. A.; Xu, H.; Nakamura, T.; Heine, T.; Chen, Q.; Jiang, D., Two-dimensional sp2 carbonconjugated covalent organic frameworks. Science 2017, 357 (6352), 673-676.

34. Unterlass, M. M.; Emmerling, F.; Antonietti, M.; Weber, J., From dense monomer salt crystals to $\mathrm{CO} 2$ selective microporous polyimides via solid-state polymerization. Chem Commun (Camb) 2014, 50 (4), 430-2.

35. Mohanrao, R.; Hema, K.; Sureshan, K. M., Topochemical synthesis of different polymorphs of polymers as a paradigm for tuning properties of polymers. Nat Commun 2020, 11 (1), 865.

36. Socrates, G., Infrared and Raman Characteristic Group Frequencies: Tables and Charts. 3 ed.; Wiley: 2004.

37. Bunck, D. N.; Dichtel, W. R., Bulk synthesis of exfoliated twodimensional polymers using hydrazone-linked covalent organic frameworks. J. Am. Chem. Soc. 2013, 135, 14952-14955.

38. Zeugolis, D. I.; Paul, R. G.; Attenburrow, G., The influence of a natural cross-linking agent (Myrica rubra) on the properties of extruded collagen fibres for tissue engineering applications. Materials Science and Engineering: C 2010, 30 (1), 190-195.

39. De Santis, R.; Sarracino, F.; Mollica, F.; Netti, P. A.; Ambrosio, L.; Nicolais, L., Continuous fibre reinforced polymers as connective tissue replacement. Composites Science and Technology 2004, 64 (6), 861-871.

40. Pharr, G. M.; Oliver, W. C., Measurement of Thin Film Mechanical Properties Using Nanoindentation. MRS Bulletin 2013, 17 (7), 28-33.

41. Kitagawa, T.; Yabuki, K.; Young, R. J., An investigation into the relationship between processing, structure and properties for high-modulus $\mathrm{PBO}$ 
fibres. Part 1. Raman band shifts and broadening in tension and compression. Polymer 2001, 42, 2101-2112.

42. Chae, H. G.; Kumar, S., Rigid-rod polymeric fibers. Journal of Applied Polymer Science 2006, 100 (1), 791-802.

43. Holmes, G. A.; Kim, J.H.; McDonough, W. G.; Riley, M. A.; Rice, K. D., A detailed investigation of the mechanical properties of polybenzoxazole fibers within soft body armor. Journal of Materials Science 2009, 44 (14), 3619-3625.

44. Imai, Y.; Itoya, K.; Kakimoto, M.-a., Synthesis of aromatic polybenzoxazoles by silylation method and their thermal and mechanical properties. Macromol. Chem. Phys. 2000, 201 (17), 2251-2256.

$45 . \quad \mathrm{Wu}, \mathrm{G}$. M.; Hung, C. H., Mechanical properties and thermo-gravimetric analysis of $\mathrm{PBO}$ thin films. Journal of Achievements in Materials and Manufacturing Engineering 2006, 17 (1-2), 27-32.

46. Fukumaru, T.; Fujigaya, T.; Nakashima, N., Mechanical Reinforcement of Polybenzoxazole by Carbon Nanotubes through Noncovalent Functionalization. Macromolecules 2013, 46 (10), 4034-4040.

47. Wei, J.; Zhang, S.; Liu, X.; Qian, J.; Hua, J.; Li, X.; Zhuang, Q., In situ synthesis of ternary $\mathrm{BaTiO} 3 / \mathrm{MWNT} / \mathrm{PBO}$ electromagnetic microwave absorption composites with excellent mechanical properties and thermostabilities. Journal of Materials Chemistry A 2015, 3 (15), 8205-8214.

48. Zhao, W.; Kong, J.; Liu, H.; Zhuang, Q.; Gu, J.; Guo, Z., Ultra-high thermally conductive and rapid heat responsive poly(benzobisoxazole) nanocomposites with self-aligned graphene. Nanoscale 2016, 8 (48), 19984-19993.

49. Feng, H.; Ma, W.; Cui, Z.-K.; Liu, X.; Gu, J.; Lin, S.; Zhuang, Q., Core/shell-structured hyperbranched aromatic polyamide functionalized graphene nanosheets-poly(p-phenylene benzobisoxazole) nanocomposite films with improved dielectric properties and thermostability. Journal of Materials Chemistry A 2017, 5 (18), 8705-8713.

50. Chen, X.; Anthamatten, M.; Harding, D. R., Vapor Deposition and Curing of Polybenzoxazole Precursors. Macromolecules 2006, 39, 7561-7565.

51. Martin, D. C.; Thomas, E. L., Ultrastructure of Poly(pphenylenebenzobisoxazole) Fibers. Macromolecules 1991, 24, 2450-2460.

52. Alemany, L. B.; Verma, M.; Billups, W. E.; Wellington, S. L.; Shammai, M., Solid- and Solution-State Nuclear Magnetic Resonance Analyses of Ecuadorian Asphaltenes: Quantitative Solid-State Aromaticity Determination Supporting the "Island" Structural Model. Aliphatic Structural Information from Solution-State $1 \mathrm{H}$ 13C Heteronuclear Single-Quantum Coherence Experiments. Energy \& Fuels 2015, 29 (10), 6317-6329.

53. Hayashi, S.; Hayamizu, K., Chemical Shift Standards in High-Resolution Solid-State NMR (1) 13C, 29Si, 1 H Nuclei. Bull. Chem. Soc. Jpn. 1991, (64), 685687. 